Check for updates

Cite this: Chem. Sci., 2020, 11, 778

๑ All publication charges for this article have been paid for by the Royal Society of Chemistry

Received 2nd November 2019

Accepted 25th November 2019

DOI: $10.1039 / c 9 s c 05538$ j

rsc.li/chemical-science

\section{Harpagide, a natural product, promotes synaptic vesicle release as measured by nanoelectrode amperometry $\dagger$}

\author{
Yun Tang, ${ }^{a}$ Xiao-Ke Yang, ${ }^{a}$ Xin-Wei Zhang, ${ }^{a}$ Wen-Tao Wu, ${ }^{a}$ Fu-Li Zhang, ${ }^{a}$ \\ Hong Jiang, ${ }^{a}$ Yan-Ling Liu, ${ }^{a}$ Christian Amatore ${ }^{* b c}$ and Wei-Hua Huang (iD *a
}

\begin{abstract}
Parkinson's disease (PD) is a neurodegenerative disorder characterized by progressive loss of dopaminergic (DAergic) neurons and low level of dopamine (DA) in the midbrain. Recent studies suggested that some natural products can protect neurons against injury, but their role on neurotransmitter release and the underlying mechanisms remained unknown. In this work, nanoelectrode electrochemistry was used for the first time to quantify DA release inside single DAergic synapses. Our results unambiguously demonstrated that harpagide, a natural product, effectively enhances synaptic DA release and restores DA release at normal levels from injured neurons in PD model. These important protective and curative effects are shown to result from the fact that harpagide efficiently inhibits the phosphorylation and aggregation of $\alpha$-synuclein by alleviating the intracellular reactive oxygen level, being beneficial for vesicle loading and recycling. This establishes that harpagide offers promising avenues for preventive or therapeutic interventions against PD and other neurodegenerative disorders.
\end{abstract}

\section{Introduction}

Among other mechanisms, neuron-neuron communications are modulated by vesicular release of neurotransmitters inside synaptic clefts followed by their detection at receptors carried by the membrane of the target neuron. ${ }^{1,2}$ Disruptions or alteration of such essential intra-synaptic cross-talk are involved in many neurologic diseases. ${ }^{3,4}$ Parkinson's disease (PD), one of the most common neurodegenerative disorders (NDDs), is characterized by a striking loss (50-70\%) of dopaminergic (DAergic) neurons in the substantia nigra pars compacta (SNpc) and massive formations of Lewy bodies by aggregation of $\alpha$-synuclein ( $\alpha$-Syn) fibrils, ${ }^{5,6}$ causing a drastic depletion of dopamine (DA) in the striatum. ${ }^{7,8}$ The causes of PD are still poorly understood, but experimental models involving 6-hydroxydopamine (6-OHDA) established that this is one archetypal neurotoxic compound prone to induce DAergic neurodegeneration by oxidative stressmediated processes. ${ }^{9-11} 6$-OHDA induces $\alpha$-Syn phosphorylation

${ }^{a}$ Key Laboratory of Analytical Chemistry for Biology and Medicine (Ministry of Education), College of Chemistry and Molecular Sciences, Wuhan University, Wuhan 430072, China.E-mail: whhuang@whu.edu.cn

${ }^{b}$ State Key Laboratory of Physical Chemistry of Solid Surfaces, College of Chemistry and Chemical Engineering, Xiamen University, Xiamen, 361005, China. E-mail: christian.amatore@ens.fr

'PASTEUR, Departement de Chimie, Pcole Normale Superieure, PSL Research University, Sorbonne Universites, UPMC Univ. Paris 06, CNRS, 24 rue Lhomond, 75005 Paris, France

$\dagger$ Electronic supplementary information (ESI) available. See DOI: $10.1039 / \mathrm{c} 9 \mathrm{sc} 05538 \mathrm{j}$ and aggregation in Lewy bodies (LBs), ultimately resulting in neurons death and neurotransmitter depletion. ${ }^{12-14}$ Therefore, real time monitoring of DA vesicular release inside neuronal synaptic clefts at the single synapse level appears of great significance to better understand the pathophysiology of PD as well as for assessing the effect of potential drugs against PD.

To prevent of the occurrence and development of PD, several agents such as monoamine oxidase inhibitors, ${ }^{15,16}$ anti-glutamatergics, ${ }^{17,18}$ glyceraldehyde-3-phosphate dehydrogenase $(\mathrm{GAPDH}){ }^{19}$ calcium channel blockers ${ }^{20}$ and neurotrophic factors $^{21}$ (i.e. glial-derived neurotrophic factor, GDNF), ${ }^{22}$ have been proposed as potential candidates to protect the neurons. However, none has conclusively been shown to reduce neuron degeneration related to PD. Fortunately, recent studies showed that some small natural molecules can modulate neurotrophic activities by promoting neuronal polarization and regenerating injured neurons. ${ }^{23-26}$ This offers promising therapeutic avenues against neurodegenerative diseases based on such natural molecules. Harpagide, an iridoid glycoside natural molecule, is an anti-inflammatory drug acting through the significant inhibitory effect of its hydrolyzed product on inflammatory factor cyclooxygenase (COX)-2 activity. ${ }^{27-29}$ This suggests that harpagide might be able to prevent mitochondrial dysfunction, enhance mitochondrial antioxidant defense and maintain neuron survival. In addition, we recently established that its concentration gradients allow guiding axon growth and regenerating injured DAergic neurons. ${ }^{30}$ However, it is still not clear if harpagide acts at the level of neurotransmitter release in synaptic clefts. 
Electrochemistry with nanoelectrode has attracted significantly increasing attention for its unique advantages (e.g. high spatiotemporal resolution, high sensitivity, minimum damage to cells) $)^{31-36}$ in real time monitoring of molecular events at multiple-scale (cell populations, single cell and subcellular) levels. ${ }^{37-48}$ Further combining with scanning electrochemical microscopy (SECM) or scanning ion conductance microscopy (SICM) allows electrochemical and topographical imaging of neurons and detection of neurotransmitter release with super resolution. ${ }^{4-51}$ Recently, amperometry at carbon-based nanoelectrodes has demonstrated its unique advantages for monitoring in real time individual vesicular exocytotic events from single synapses or varicosities and providing statistically significant quantitative and kinetic information achievable by no other methods. ${ }^{52,53}$ In this work, extremely tiny carbon fiber nanoelectrodes with smaller size and higher spatial resolution that our previous ones were fabricated (Fig. 1A), and inserted into central (DAergic) neurons synapses to probe individual dopamine releasing events (Fig. 1B). This demonstrated that harpagide can effectively promote dopamine synaptic release and restore it at normal levels in neurons damaged by 6-OHDA. Further data involving fluorescence probes for evaluating the production of ROS incidence, as well as identifying the expression of $\alpha$-Syn monomer and phosphorylated $\alpha$-Syn (P-Syn) demonstrated that harpagide decreases intracellular ROS level and inhibits phosphorylation of $\alpha$-Syn monomers in neurons. This work discloses first direct quantitative evidence that natural molecules such as harpagide may promote and/or restore neurotransmitter release from synaptic vesicles. These data suggest a potential mechanism through which these properties are implemented, which is expected to be beneficial in developing novel therapeutic strategies for PD and other NDDs.

\section{Results and discussion}

\section{Nanoelectrode fabrication and insertion inside single synapses}

Cone-shaped carbon fiber nanoelectrodes (CFNEs) with 50$100 \mathrm{~nm}$ tip diameter and 500-2000 $\mathrm{nm}$ shaft length were fabricated (Fig. 1A, the detailed fabrication process can be found in our previous work $^{52,53}$ and also described in
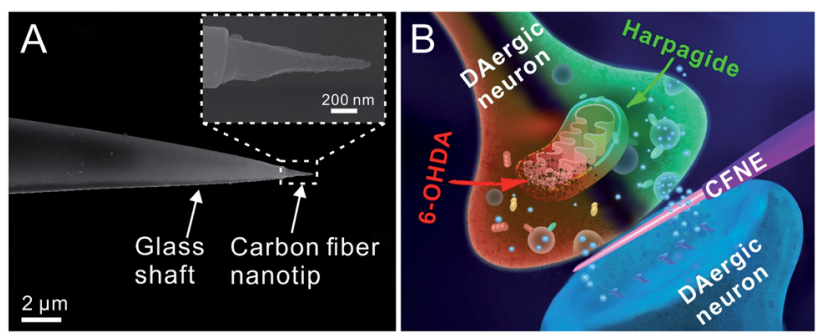

Fig. 1 (A) SEM image of a conical CFNE tip, the scale bar is $2 \mu \mathrm{m}$. Inset: A magnified SEM image of the tip, the scale bar is $200 \mathrm{~nm}$. (B) Schematic representation of a cone-shaped carbon fiber nanoelectrode inside an individual DAergic neurons synapse. methods). The excellent electrochemical characteristics of these CFNEs was tested and validated by recording steadystate cyclic voltammograms such as that displayed in Fig. S1. $\dagger$ Both the nanotip of a CFNE and the single DAergic synapse can be visualized by optical microscopy, allowing slipping the nanotip into the synaptic cleft ( $82 \%$ success rate, $n=1250$ ) without inducing any observable damage to neurons (Fig. 2A and $\mathrm{S} 2 \dagger$ ). The tight sealing of the synaptic cleft around CFNEs during their insertion process was controlled by adding $\left[\mathrm{Ru}\left(\mathrm{NH}_{3}\right)_{6}\right]^{3+}$, a biocompatible ionic species, to the culture medium and recording the electrochemical reduction current signal of $\left[\mathrm{Ru}\left(\mathrm{NH}_{3}\right)_{6}\right]^{3+}$ at different insertion depths inside the synaptic cleft (Fig. 2B). The diffusion-limited $\left[\mathrm{Ru}\left(\mathrm{NH}_{3}\right)_{6}\right]^{3+}$ reduction currents dropped to $15 \%(n=4)$ of their original values after complete insertion, while they were almost fully restored $(>90 \%, n=4)$ after complete withdrawal from the synaptic cleft. Altogether these tests assure that in the next experiments any neurotransmitter fluxes arose from within the synapses and the insertion process did not affect the sensitivity of CFNEs.

\section{Harpagide promotes DA release in primary DAergic neurons}

Intrasynaptic individual dopamine release events were monitored through the oxidation of dopamine ${ }^{54}$ at the tip of CFNEs inserted inside single synapses formed by DAergic neurons cultured under normal conditions (DEM/F12, Fig. S3(i) $\dagger$ ), and whose amperometric responses (Fig. 3A(i)) were used as controls in this study. DA release was elicited by brief $\mathrm{K}^{+}(62.5$ $\mathrm{mM}$ ) pulses leading to a series of amperometric spikes corresponding to sequential vesicular release from single synaptic vesicles. Statistical analyses of the recorded individual current spikes (superimposed spikes were excluded as well as complex ones $^{52}$ ) showed that the individual peak currents $\left(I_{\max }\right)$ and peak area $(Q$, being proportional to the number $N$ of released molecules according to Faraday's law, ${ }^{54} Q=2 F N$ where $F=96500 \mathrm{C}$ is the Faraday constant) followed normal distributions (Fig. 3B, $\mathrm{C}$ and $\mathrm{S} 4 \dagger$ ) with $I_{\max }=18.1 \pm 0.6 \mathrm{pA}$, and $N=13800 \pm 5100$ molecules (mean \pm S.E.M.; $n=808$ ).
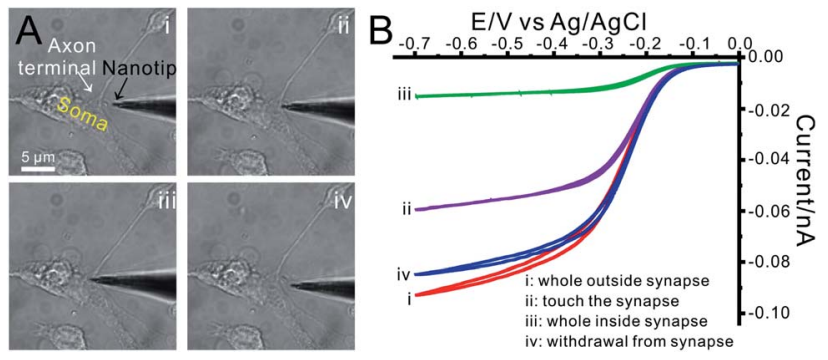

Fig. 2 (A) Bright-field photomicrographs displaying the insertion of a nanoelectrode tip inside a synaptic cleft between a DAergic neuron axon terminal and another DAergic neuron soma; snapshots (i-iii) represents the sequential insertion stages while (iv) features the same CFNE after its withdrawing from the synapse. (B) Steady state cyclic voltammograms $\left(0.1 \mathrm{~V} \mathrm{~s}^{-1}\right)$ recorded at different penetration depths of a conical CFNE nanotip into a synapse when $1 \mathrm{mM}\left[\mathrm{Ru}\left(\mathrm{NH}_{3}\right)_{6}\right]^{2+/ 3+}$ was added to the extracellular medium. 

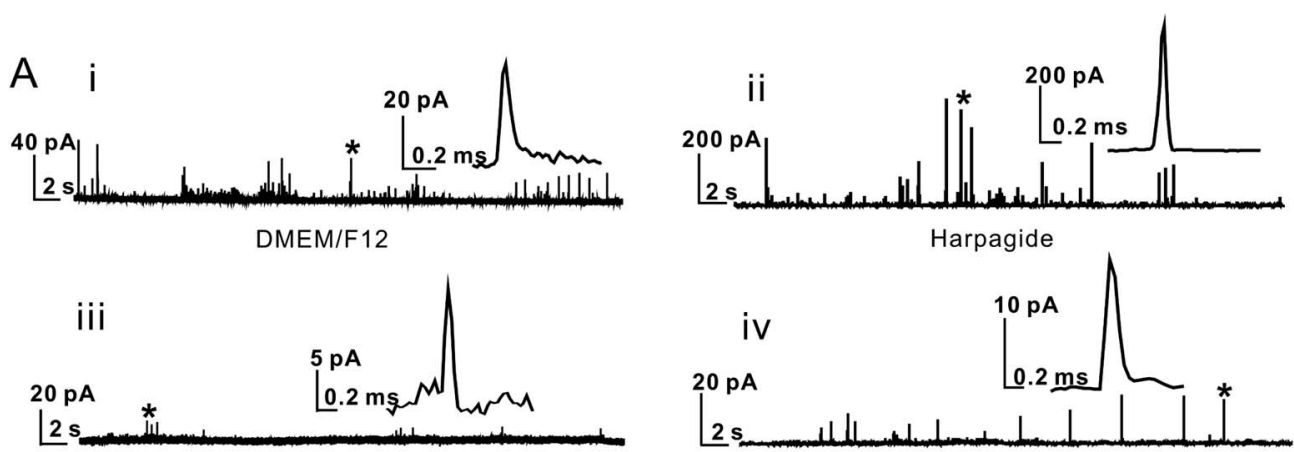

6-OHDA

Harpagide + 6-OHDA
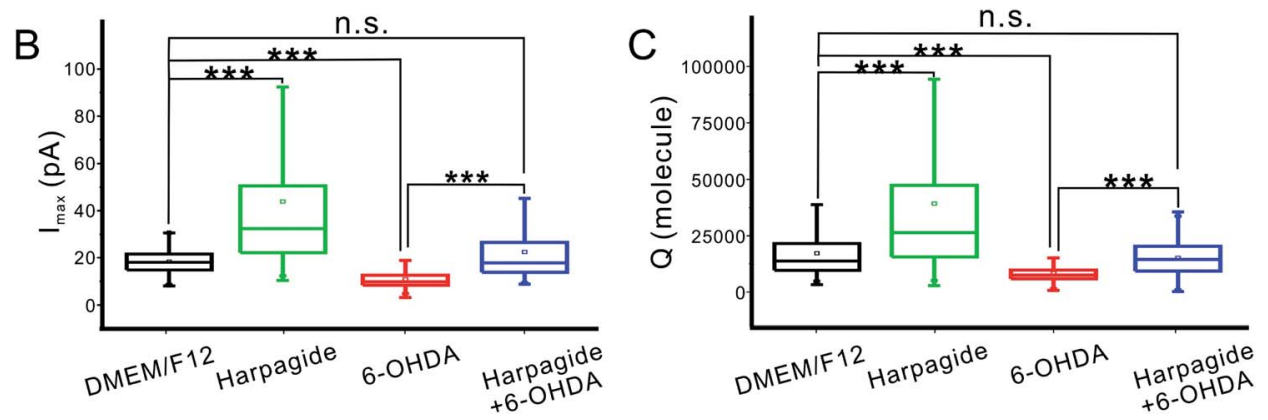

Fig. 3 (A) Representative amperometric traces recorded from $\mathrm{K}^{+}$-elicited $(62.5 \mathrm{mM})$ exocytotic release recorded with a conical CFNE inserted into the synaptic clefts of untreated neurons (i), harpagide-treated neurons (ii), 6-OHDA-treated neurons (iii) and pre-treated with harpagide before exposure to 6-OHDA (iv); in each amperometric trace one event marked by asterisk is shown using enlarged scales. (B and C) Statistical analyses of the average current amplitude $I_{\max }$ and the number of molecules of dopamine per vesicle (as given by the spike charge amplitude $Q$ ) under the four different situations investigated here, and the number of events was 808, 904, 390 and 575 for each group, respectively; (one-way ANOVA; ***p $\leq 0.001 ;$ n.s.: not significant).

Pretreating the DAergic neurons with $10 \mu \mathrm{M}$ harpagide (Fig. S3(ii)†) added to the normal culture medium led to the recording of amperometric spikes with larger amplitude (Fig. 3A(ii)). Statistical analyses showed that $I_{\max }$ and $Q$ obeyed slanted normal distributions with $I_{\max }=32.3 \pm 1.6 \mathrm{pA}$, and $N=$ $26300 \pm 15000$ (Fig. 3B, C and S4; †n=904). Since harpagide is not an electroactive molecule (Fig. S6†), this demonstrates that it plays a biological role that results in significantly enhancing neurotransmitter vesicular release from DAergic neurons (see below for the exocytotic frequency).

\section{Harpagide maintains DA release at normal level in a cell model of PD}

To evaluate the neuroprotective effect of harpagide, a PD-cell model was created by pretreating the DAergic neurons with a neurotoxic agent, 6-hydroxydopa-mine (6-OHDA), and individual dopamine synaptic release events monitored amperometrically as performed under control conditions (Fig. S3(iii): see $\mathrm{ESI} \uparrow$ for the optimization of 6-OHDA concentration). Only a few small amperometry spikes could be detected for 6-OHDAtreated DAergic neurons (Fig. 3A(iii)) giving rise to near normal distributions (Fig. S7 $\dagger$ ) of $I_{\max }$ and $Q$ values (Fig. 3B, C and S6 $\dagger$ ) with $I_{\max }=9.8 \pm 0.2 \mathrm{pA}$ and $N=7500 \pm 200$ molecules $(n=$ 390). This confirmed that 6-OHDA induced strong damages to DAergic neurons and drastically altered their synaptic functions by (i) severely reducing the number of vesicular events (see below for the exocytotic frequency) and (ii) reducing by $c a$. $55 \%$ the amount of DA released during the very few events which could be monitored.

Conversely, when the neurons were pre-incubated with harpagide before the 6-OHDA treatment (Fig. S3(iv) $\dagger$ ), the number of amperometric spikes per trace was similar to that of control measurements (Fig. 3A(iv)), and the spikes displayed characteristics almost identical to those of untreated neurons: $I_{\max }=$ $17.9 \pm 0.3 \mathrm{pA}$ and $N=12700 \pm 900$ molecules (Fig. 3B, C and S6; $\dagger n=575)$. This demonstrates that pre-incubation with harpagide can efficiently protect DAergic neurons synaptic functions and maintain vesicle exocytosis at the normal level (see below for the exocytotic frequency).

\section{Harpagide suppresses the $\alpha$-Syn phosphorylation in primary DAergic neurons by inhibiting ROS production}

6-OHDA has been reported to induce mitochondria dysfunction via the generation of hydrogen peroxide and derived hydroxyl radicals which induce $\alpha$-Syn phosphorylation in DAergic neurons further resulting in the progressive degeneration of DAergic neurons..$^{9,12,13,55-57}$ Conversely, harpagide was suggested to possess anti-inflammatory effects reducing the overproduction of cellular peroxides ${ }^{27-29}$ in agreement with our observations. To confirm that the neuro-promotive and neuroprotective effects of harpagide on DAergic neurons are linked to these reported properties, the intracellular ROS content was 
A

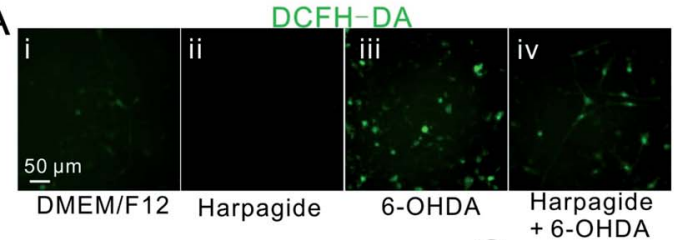

C

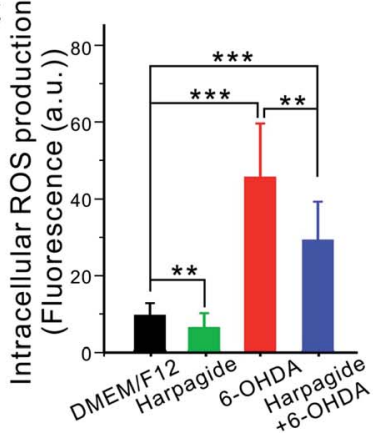

$\mathrm{D} \stackrel{0}{\circ}$ +6-OHDA

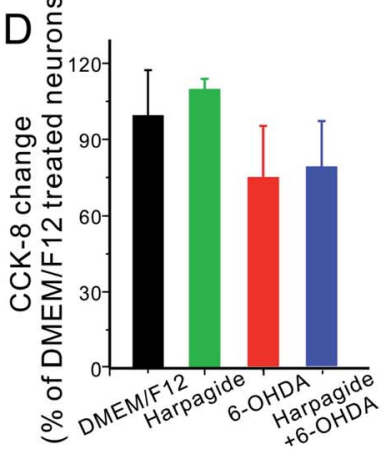

a-Syn/Hoechst
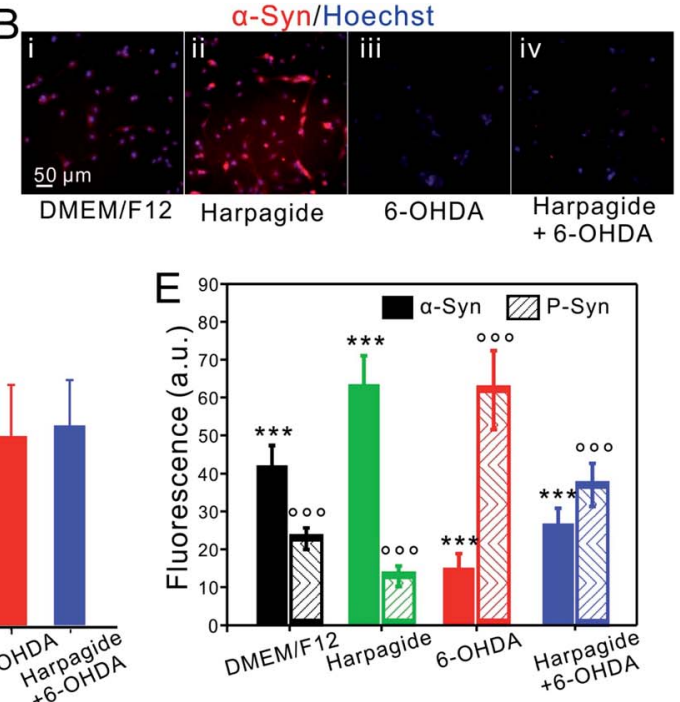

Fig. 4 (A) Representative microscopic images of the intracellular ROS content revealed by DCFH-DA green fluorescence in DAergic neurons cultured under different conditions (i) controls; (ii) pre-treated with harpagide; (iii) pretreated with 6-OHDA; and (iv) pre-treated with 6-OHDA after being incubated with harpagide. (B) Same as in (A), with the red fluorescence characterizing the presence of $\alpha$-synuclein ( $\alpha$-Syn); the blue fluorescence indicates the nucleus of neurons (Hoechst fluorescent test for cell nucleus). (C-E) Quantitative analyses of the intracellular ROS level (C), DAergic neurons activity (D), $\alpha$-Syn and P-Syn expression in each of the four cell groups (E). Scale bars in (A and B) $50 \mu \mathrm{m}$ (one-way ANOVA $* * p \leq 0.01, * * * p \leq 0.001$ and $\left.{ }^{\circ 00} p \leq 0.001\right)$

evaluated with a ROS fluorescent assay kit based on $2^{\prime}, 7^{\prime}$ dichloro-dihydro-fluorescein diacetate, DCFH-DA. As shown in Fig. 4A, C and S7, $\dagger$ the intracellular ROS levels in control DAergic neurons were significantly decreased when pre-treated with harpagide or drastically increased by pre-treating them with 6-OHDA. In agreement with the amperometric results, the ROS elevation induced by 6-OHDA was efficiently inhibited when the DAergic neurons were pre-incubated with harpagide. Similar conclusions were drawn when performing a cell viability assay (Cell Counting Kit-8, CCK-8) (Fig. 4E and S7 $\dagger$ ) or by statistical analysis of the neuronal axon lengths (Fig. S8 + ). Altogether, these results demonstrate that harpagide can reduce ROS intracellular generation, enhance neural activity and promote neuronal growth.

$\alpha$-Syn is highly enriched in pre-synaptic neuron buttons, being implicated in the modulation of synaptic activity through regulating synaptic vesicle release. ${ }^{58,59}$ However, its phosphorylation into P-Syn following its ROS-induced modification at Ser129 (pSer129) caused by mitochondrial dysfunction in PD was shown to disrupt this regulation ${ }^{59,60}$ in agreement with our above results. The intracellular contents of $\alpha$-Syn and P-Syn were thus assessed based on immunofluorescent assays. The results shown in Fig. 4B, E, S9 and $\mathrm{S} 10^{\dagger} \dagger$ demonstrate that harpagide-pretreated DA neurons display higher concentrations of $\alpha$-Syn but lower ones of P-Syn than that in control neurons, while 6-OHDA-treatment caused a significant decrease of $\alpha$-Syn correlated with a higher expression of P-Syn; conversely, the higher expression of P-Syn caused by 6-OHDA was effectively inhibited when DAergic neurons were pre-incubated with harpagide. These results are in total agreement with the amperometric ones reported above, suggesting that harpagide could act beneficially on the synaptic activity by suppressing or avoiding the ROS-induced $\alpha$-Syn phosphorylation so as to maintain or restore the dynamic $\alpha$-Syn/P-Syn balance inside neurons (Fig. S11†).

\section{Harpagide promotes the frequency of exocytosis by increasing} the total amount of presynaptic exocytotic vesicles

Recent studies suggested that $\alpha$-Syn also enhances vesicle membrane stabilization and promote synaptic vesicle recycling, whereas P-Syn has the converse effect. ${ }^{59-61}$ Our amperometric results fully support these statements on quantitative grounds. Indeed, in addition to enhancing the average number of released molecules per exocytotic spike (Fig. 3C and D), harpagide also increases the frequency of amperometric spikes. Statistical analyses showed that in any given time period $(60 \mathrm{~s})$, $99 \pm 4$ current spikes per min were detected in DAergic control neurons. This frequency increased by $c a$. 20\% (118 \pm 5 spikes per min) when the neurons were pre-incubated with harpagide solution (Fig. 3B and 5A). Conversely, 6-OHDA drastically reduced the synaptic vesicle exocytosis frequency at $c a .35 \%$ of controls ( $35 \pm 1$ spikes per min), but only to $c a .50 \%$ of controls ( $48 \pm 1$ spikes per $\min$ ) when the DAergic neurons were incubated with harpagide before 6-OHDA (Fig. 3B and 5A).

Synaptophysin is an integral glycoprotein localized in synaptic vesicles without apparent role on exocytosis but that may be used for quantifying the density of presynaptic vesicles by immunofluorescent staining. ${ }^{62}$ As shown in Fig. 5B and C, the number of vesicles was obviously increased in harpagidepretreated neurons compared with control neurons, while 6OHDA injury caused a significant decline in synaptic vesicle number. In agreement with all the above results, the 6-OHDA 

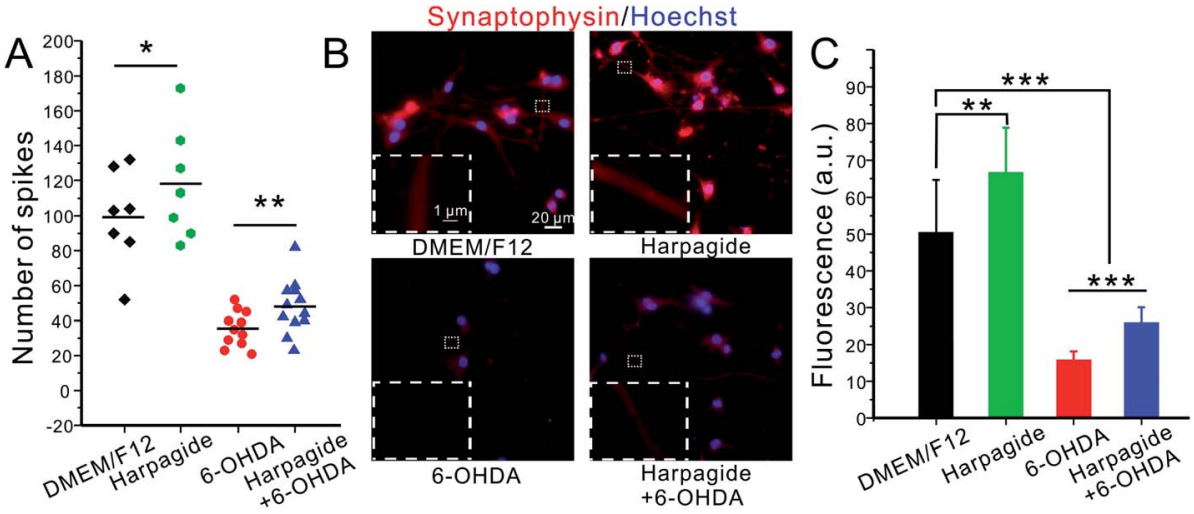

Fig. 5 (A) Scatter plot showing the distribution of amperometric spikes numbers detected during a 60 s time period; black symbols: untreated controls; green symbols: harpagide-pretreated neurons; red symbols: 6-OHDA-injured neurons; blue symbols: neurons pretreated with harpagide before 6-OHDA incubation. (B) Representative microscopic views for each above group of immunostained neurons (the common markers in each panel and inset represent $20 \mu \mathrm{m}$ and $1 \mu \mathrm{m}$, respectively); the red fluorescence features the synaptophysin presence, and the blue (Hoechst) one indicates the nucleus of neurons; insets: amplified views $(\times 100)$ of single axon under each situation. (C) Corresponding statistical analysis of the data in $(\mathrm{B}) ; * * p \leq 0.01 ; * * * p \leq 0.001$.

induced decline was significantly inhibited by pre-incubation with harpagide. This is fully coherent with the different relative frequencies of amperometric individual events monitored by amperometry under the four situations investigated here and stress the neuroprotective role of harpagide in maintaining the stability of exocytotic vesicles and synaptic functions against ROS-induced alterations.

\section{Conclusions}

In summary, carbon-based nanoelectrodes were developed and used for direct amperometric monitoring of individual vesicular exocytotic events occurring inside individual synapses. This was applied to evaluate the purported neuroprotective properties of harpagide, a natural product, against alterations incurred by DAergic neurons in PD-models. Amperometric intrasynaptic measurements afforded for the first time quantitative data to characterize these neuro-stimulating and neuroprotective effects. Our results strongly establish that harpagide promotes vesicular synaptic function, hence leading to increased release, through inhibition of ROS-induced phosphorylation of $\alpha$-Syn, and that harpagide efficiently protects DAergic neurons from being injured by significant ROS fluxes generated by important ROS-promoters such as 6-OHDA (Fig. S12†). Though the generality of this mechanism should be further assessed in other kinds of neurons, this work provides new directions for designing preventive or therapeutic strategies for PD and other NDDs based on small anti-inflammatory molecules.

\section{Experimental}

\section{Materials}

DMEM/F12 and B27 GIBCO ${ }^{\circledR}$ (U.S.A.) and trypsin (no. 0458) was bought from Amresco (U.S.A.). Poly-L-lysine (PLL), laminin and nerve growth factor (NGF) were purchased form Sigma (St. Louis, MO).
Harpagide was obtained from Hubei Jusheng Technology Co., Ltd (Wuhan, China). Anti- $\alpha$-synuclein antibody, anti- $\alpha$-synuclein (phospho S129) antibody and anti-synaptophysin antibody were purchased from Abcam (U.K.). Anti-tyrosine hydroxylase (TH) was purchased from and the fluorescent secondary antibodies were purchased from Wuhan Boster Corp (China). Reactive oxygen species (ROS) assay kit and cell counting kit-8 (CCK-8) assay were purchased from Beyotime Biotechnology Ltd. (China) and Dojindo Laboratories (Japan), respectively. All other chemicals unless specified were reagent grade and were used without further purification.

\section{Nanoelectrode fabrication}

The carbon fiber nanoelectrodes (CFNEs) were fabricated as previously reported..$^{52,53}$ Briefly, a glass capillary (1B100F-4, Word Precision Instruments) was pulled to form a submicropipette under a laser micropuller (P2000, Sutter Instruments, U.S.A.), and a $7 \mu \mathrm{m}$ diameter carbon fiber (Goodfellow Co., Oxford, U.K.) was flame etched to form a needle-shape nanotip. The etched carbon fiber was then inserted into the sub-micropipette until several micrometers protruded. The tip of the carbon fiber was then fused inside the sub-micropipette and further etched by the heated platinum wire $(25 \mu \mathrm{m}$ diameter) of a microforge (MF900, World Precision Instruments, USA) to form a conical carbon fiber nanoelectrode with the diameter ranged from $50-100 \mathrm{~nm}$ (Fig. 1A). The electrochemical characterization (Fig. S1 $\dagger$ ) was performed using an electrochemical workstation (CHI 660A, CH Instruments, Shanghai, China), and the SEM characterization was performed on a scanning electron microscope (FE-SEM, SIGMA, Zeiss, Germany).

\section{DA neuronal culture}

Primary DA neurons were isolated from a one-day-old SD rat, and the ventral tegmental area (VTA) were dissociated using a previously described protocol. ${ }^{30,63}$ In brief, the VTA was 
dissected from the mesencephalon of a one-day-old SD rat, which mainly comprised DA neurons and neuroglial cells. This preparation was incubated with $0.125 \%$ trypsin at $37^{\circ} \mathrm{C}$ in a $\mathrm{CO}_{2}$ incubator for $3 \mathrm{~min}$ before adding the same volume of serumfree culture medium to terminate the action of the trypsin. After $1 \mathrm{~h}$, in order for the neurons to attach to the culture dish, serum-free primary culture medium of DMEM/F12 was transferred to inhibit the growth of neuroglial cells and then supplemented with $2 \% \mathrm{~B} 27^{\mathrm{TM}}$ and $100 \mathrm{U} \mathrm{mL}^{-1}$ penicillin and streptomycin. A low concentration of NGF $\left(10 \mathrm{ng} \mathrm{mL}^{-1}\right)$ was added to the medium to favor neuron survival. Finally, the culture dishes were placed in an incubator at $37^{\circ} \mathrm{C}$ and $5 \% \mathrm{CO}_{2}$ for neuronal culture.

\section{Exocytosis detection and data analysis}

As reported in Fig. 2A and S2, $\uparrow$ the nanotip of a CFNE was positioned with a micromanipulator (Transfer Man NK2, Eppendorf, Hamburg, Germany) under close observation by inverted microscopy using $40 \times$ objective lens. When a synapse was successfully identified under the microscope, the tip of the nanoelectrode was firstly located nearby the intersection of the single varicosity, and then was slowly wedged into the synapse formed by the varicosity and its target (the soma) till a slight distortion of the synapse occurred with a micromanipulator (Fig. 2A and S2 $\dagger$ ).To evoke exocytosis, a pulse of $62.5 \mathrm{mM}-\mathrm{K}^{+}$ solution was delivered via a glass capillary connected to an injection pump placed about $50 \mu \mathrm{m}$ away from the probed synaptic cleft using another micromanipulator. The amperometric spikes were recorded with a patch clamp amplifier (EPC10, HEKE Electronics) at a constant potential of $+750 \mathrm{mV}^{52,53}$ with an $\mathrm{Ag} / \mathrm{AgCl}$ as the reference/counter electrode. Signals were sampled at $20 \mathrm{kHz}$, and raw amperometric data were collected using "pulse" software, and analyzed according to previously described method ${ }^{52,53,64}$ using a routine in Igor Pro (Igor Pro, Wave Metrics) kindly provided by Dr E. V. Mosharow in Columbia University. $I_{\max }$ and $Q$ values were recorded for each current spike and statistically analyzed provided that the $I_{\max }$ value was larger than 6 -fold standard deviation of the background noise. Superimposed current spikes and/or spikes with complex shapes were discarded to avoid unwanted interferences.

\section{CCK-8 assay}

Petri culture dishes covered with cultured DAergic neurons were gently rinsed with PBS and then $1 \mathrm{ml}$ of DMEM/F12 containing $10 \%$ CCK- 8 was added in the Petri dish before incubation at $37^{\circ} \mathrm{C}$ for $2 \mathrm{~h}$. The supernatant was then transferred to a 96-well plate and the optical density (O.D.) measured at $450 \mathrm{~nm}$ using an ELX808 Ultra Microplate Reader (Bio-Tek Instruments, Inc., America).

\section{Intracellular ROS measurements}

The ROS assay kit (DCFH-DA) solution was added according to the manufacturer's protocol to the medium containing DAergic neurons prepared under different conditions (Fig. 3A). After incubation at $37^{\circ} \mathrm{C}$ for $20 \mathrm{~min}$, the residual dye was washed off with PBS to ensure that the green fluorescence arose exclusively from the $2^{\prime}, 7^{\prime}$-dichlorofluorescin diacetate (DCFH-DA) probe that had reacted with ROS. Bright field and fluorescence microphotographs of the cell cultures were recorded on an inverted fluorescent microscopy ( $40 \times$ objective, Zeiss Observer Z1, Carl Zeiss, Germany). During fluorescent analyses, the exciting light intensity and exposure time were kept identical to ensure that the microphotographs recorded from each batch one realistically represented the intracellular ROS concentrations.

\section{Immunofluorescence}

DAergic neurons cultures were rinsed 3 times with $0.1 \mathrm{M}$ PBS and fixed for $30 \mathrm{~min}$ in $4 \%$ paraformaldehyde (in $0.1 \mathrm{M}$ PBS). The neurons were then permeabilized by a $30 \mathrm{~min}$ treatment in $0.2 \%$ Triton X-100 (in $0.1 \mathrm{M}$ PBS), and blocked for $30 \mathrm{~min}$ in $1.5 \%$ normal goat serum (NGS, in $0.1 \mathrm{M}$ PBS). To label $\alpha$-Syn, PSyn and synaptophysin, neurons were incubated with the corresponding primary antibodies (rabbit, 1:200) overnight at $4{ }^{\circ} \mathrm{C}$, then washed with $0.1 \mathrm{M}$ PBS and incubated with a TRITClabeled secondary antibody (rat anti-rabbit IgG TRITC, 1:100) for $1 \mathrm{~h}$ at $37^{\circ} \mathrm{C}$, respectively. Immunofluorescence location and intensity was visualized with a Zeiss Observer Z1 inverted fluorescent microscope with appropriate fluorescent filters.

\section{Image analysis and statistics}

The length of the axons was traced and quantified using an ImageJ plug-in (NeuronJ) software. The fluorescence intensity was also quantified using an ImageJ. SPSS 19.0 (SPSS Inc.) was used to perform statistical analysis (one-way ANOVA). The error bars are standard errors of the mean, $n \geq 3$.

\section{Conflicts of interest}

There are no conflicts to declare.

\section{Acknowledgements}

We gratefully acknowledge financial support from the National Natural Science Foundation of China (Grants 21725504, 21675121, and 21721005), the University of Xiamen and the State Key Laboratory of Physical Chemistry of Solid Surfaces (PCOSS, University of Xiamen, China) through a Distinguished Scientist Chair awarded to C.A. In France, it was supported in part by PSL University, École Normale Supérieure, CNRS, and Sorbonne University (UMR 8640). Both teams gratefully acknowledge the CNRS Sino-French support (LIA "NanoBioCatEchem").

\section{References}

1 T. Branco and K. Staras, Nat. Rev. Neurosci., 2009, 10, 373383.

2 C. F. Stevens, Neuron, 2003, 40, 381-388.

3 O. Marín, Nat. Rev. Neurosci., 2012, 13, 107-120.

4 A. Sawa and S. H. Snyder, Science, 2002, 296, 692-695. 
5 C. A. Davie, Br. Med. Bull., 2008, 86, 109-127.

6 K. C. Luk, C. Song, P. O'Brien, A. Stieber, J. R. Branch, K. R. Brunden, J. Q. Trojanowski and V. M. Y. Lee, Proc. Natl. Acad. Sci. U. S. A., 2009, 106, 20051-20056.

7 M. Carli, J. L. Evenden and T. W. Robbins, Nature, 1985, 313, 679-682.

8 R. B. Innis, J. P. Seibyl, M. Laruelle, A. A. Dargham, E. Wallace, R. M. Baldwin, Y. Z. Ponce, S. Zoghbi and S. Wang, Proc. Natl. Acad. Sci. U. S. A., 1993, 90, 11965-11969.

9 D. Blum, S. Torch, N. Lambeng, M. F. Nissou, A. L. Benabid, R. Sadoul and J. M. Verna, Prog. Neurobiol., 2001, 65, 135172.

10 T. Kim, Cold Spring Harbor Perspect. Med., 2011, 1, a009316. 11 S. Andreas, Cell Tissue Res., 2004, 318, 215-224.

12 C. W. Yvette and K. Dimitri, Nat. Med., 2017, 23, 1-13.

13 O. S. Gorbatyuk, S. Li, L. F. Sullivan, W. J. Chen, G. Kondrikova, F. P. Manfresson, R. J. Mandel and N. Muzyczka, Proc. Natl. Acad. Sci. U. S. A., 2008, 105, 763768.

14 L. Julie and B. Patrik, Nat. Rev. Neurosci., 2002, 12, 932-942. 15 M. B. H. Youdim, D. Edmondson and K. F. Tipton, Nat. Rev. Neurosci., 2006, 4, 295-309.

16 M. Thomas and D. M. Jan, Expert Opin. Drug Metab. Toxicol., 2019, 5, 429-435.

17 E. Igor and B. David, Neuron, 2016, 89, 11-36.

18 K. M. Sumeer and E. Clare, Behav. Neurol., 2013, 26, 255-263.

19 M. R. Hara, B. Thomas, M. B. Cascio, B. Bae, L. D. Hester, V. L. Dawson, T. M. Dawson, A. Sawa and S. H. Snyder, Proc. Natl. Acad. Sci. U. S. A., 2006, 103, 3887-3889.

20 C. S. Chan, J. N. Guzman, E. Ilijic, J. N. Mercer, C. Rick, T. Tkatch, G. E. Meredith and D. J. Surmeier, Nature, 2007, 44, 1081-1086.

21 M. A. Caldwell, X. L. He, N. Wilkie, S. Pollack, G. Marshall, K. A. Wafford and C. N. Svendsen, Nat. Biotechnol., 2001, 19, 475-479.

22 K. Deniz, G. Biljana and B. Anders, Nat. Neurosci., 2004, 7, 105-110.

23 X. Y. Cheng and G. C. Micalizio, J. Am. Chem. Soc., 2016, 138, 1150-1153.

24 J. Xu, M. H. Lacoske and E. A. Theodorakis, Angew. Chem., Int. Ed., 2014, 53, 956-987.

25 M. Alghazwi, Y. Q. Kan, W. Zhang, W. P. Gai, M. J. Garson and S. Smid, J. Appl. Phycol., 2016, 28, 3599-3616.

26 R. Tundis, M. Loizzo, F. Menichini, G. A. Statti and F. Menichini, Mini-Rev. Med. Chem., 2008, 8, 399-420.

27 V. Gyurkovska, K. Alipieva, A. Maciuk, P. Dimitrova, N. Ivanovska, C. Haas, T. Bley and M. Georgiev, Food Chem., 2011, 125, 171-178.

28 A. Viljoen, N. Mncwangi and I. Vermaak, Curr. Med. Chem., 2012, 19, 2104-2127.

29 L. Q. Zhang, L. Feng, Q. Jia, J. W. Xu, R. Wang, Z. T. Wang, Y. C. Wu and Y. M. Li, Bioorg. Med. Chem., 2011, 19, 48824886.

30 Y. Tang, Q. F. Qiu, F. L. Zhang, M. Xie and W. H. Huang, Lab Chip, 2018, 18, 971-978.

31 Y. S. Fan, C. Han and B. Zhang, Analyst, 2016, 141, 54745487.
32 J. Clausmeyer and W. Schuhmann, TrAC, Trends Anal. Chem., 2016, 79, 46-59.

33 N. T. N. Phan, X. C. Li and A. G. Ewing, Nat. Rev. Chem., 2017, 1, 1-18.

34 Y. L. Ying, Z. F. Ding, D. P. Zhan and Y. T. Long, Chem. Sci., 2017, 8, 3338-3348.

35 J. Y. Zhou, D. C. Jiang and H. Y. Chen, Sci. China: Chem., 2017, 60, 1277-1284.

36 L. M. Zhang and Y. Tian, Acc. Chem. Res., 2018, 51, 688-696.

37 D. S. Kim, E. S. Kang, S. Baek, S. S. Choo, Y. H. Chung, D. Lee, J. Min and T. H. Kim, Sci. Rep., 2018, 8, 14049.

38 T. H. Kim, C. H. Yea, S. T. D. Chueng, P. T. T. Yin, B. Conley, K. Dardir, Y. Pak, G. Y. Jung, J. W. Choi and K. B. Lee, Adv. Mater., 2015, 27, 6356-6362.

39 P. Actis, S. Tokar, J. Clausmeyer, B. Babakinejad, S. Mikhaleva, R. Cornut, Y. Takahashi, A. López Córdoba, P. Novak, A. I. Shevchuck, J. A. Dougan, S. G. Kazarian, P. V. Gorelkin, A. S. Erofeev, I. V. Yaminsky, P. R. Unwin, W. Schuhmann, D. Klenerman, D. A. Rusakov, E. V. Sviderskaya and Y. E. Korchev, ACS Nano, 2014, 8, 875-884.

40 X. C. Li, S. Majdi, J. Dunevall, H. Fathali and A. G. Ewing, Angew. Chem., Int. Ed., 2015, 54, 11978-11982.

41 Y. Z. Liu, M. Li, F. Zhang, A. W. Zhu and G. Y. Shi, Anal. Chem., 2015, 87, 5531-5538.

42 R. R. Pan, M. C. Xu, D. C. Jiang, J. D. Burgess and H. Y. Chen, Proc. Natl. Acad. Sci. U. S. A., 2016, 113, 11436-11440.

43 L. Ren, M. D. Pour, S. Majdi, X. C. Li, P. Malmberg and A. G. Ewing, Angew. Chem., Int. Ed., 2017, 56, 4970-4975.

44 Y. Li, K. K. Hu, Y. Yu, S. A. Rotenberg, C. Amatore and M. V. Mirkin, J. Am. Chem. Soc., 2017, 139, 13055-13062.

45 X. W. Zhang, Q. F. Qiu, H. Jiang, F. L. Zhang, Y. L. Liu, C. Amatore and W. H. Huang, Angew. Chem., Int. Ed., 2017, 56, 12997-13000.

46 Y. L. Ying, Y. X. Hu, R. Gao, R. J. Yu, Z. Gu, L. P. Lee and Y. T. Long, J. Am. Chem. Soc., 2018, 140, 5385-5392.

47 R. R. Pan, M. C. Xu, J. D. Burgess, D. C. Jiang and H. Y. Chen, Proc. Natl. Acad. Sci. U. S. A., 2018, 115, 4087-4092.

48 K. K. Hu, Y. Li, S. A. Rotenberg, C. Amatore and M. V. Mirkin, J. Am. Chem. Soc., 2019, 141, 4564-4568.

49 Y. Takahashi, A. I. Shevchuk, P. Novak, Y. J. Zhang, N. Ebejer, J. V. Macpherson, P. R. Unwin, A. J. Pollard, D. Roy, C. A. Clifford, H. Shiku, T. Matsue, D. Klenerman and Y. E. Korchev, Angew. Chem., Int. Ed., 2011, 50, 9638-9642.

50 M. Shen, Z. Z. Qu, J. DesLaurier, T. M. Welle, J. V. Sweedler and R. Chen, J. Am. Chem. Soc., 2018, 140, 7764-7768.

51 F. P. Filice and Z. F. Ding, Analyst, 2019, 144, 738-752.

52 Y. T. Li, S. H. Zhang, L. Wang, R. R. Xiao, W. Liu, X. W. Zhang, Z. Zhou, C. Amatore and W. H. Huang, Angew. Chem., Int. Ed., 2014, 53, 12456-12460.

53 Y. T. Li, S. H. Zhang, X. Y. Wang, X. W. Zhang, A. I. Oleinick, I. Svir, C. Amatore and W. H. Huang, Angew. Chem., Int. Ed., 2015, 54, 9313-9318.

54 E. L. Ciolkowski, K. M. Maness, P. S. Cahill, R. M. Wightman, D. H. Evans, B. Fosset and C. Amatore, Anal. Chem., 1994, 66, 3611-3617. 
55 S. H. Guo, J. Q. Yan, X. Q. Yang, E. Bezard and B. L. Zhao, Psychiatry, 2007, 62, 1353-1362.

56 Y. C. Shen, Y. J. Shiao, Y. J. Sung and C. N. Wang, J. Chin. Med., 2005, 16, 63-87.

57 J. Lautenschläger, C. F. Kaminski and G. S. K. Schierle, Trends Cell Biol., 2017, 27, 468-479.

58 J. S. Dennis, Nat. Neurosci., 2017, 20, 629-631.

59 K. Vekrellis, M. Xilouri, E. Emmanouilidou, H. J. Rideout and L. Stefanis, Lancet Neurol., 2011, 10, 1015-1025.
60 V. M. Nemani, W. Lu, V. Berge, K. Nakamura, B. Onoa, M. K. Lee, F. A. Chaudhry, R. A. Nicoll and R. H. Edwards, Neuron, 2010, 65, 66-79.

61 A. Pineda and J. Burré, Proc. Natl. Acad. Sci. U. S. A., 2017, 114, 1223-1225.

62 T. Söllner, M. K. Bennett, S. W. Whiteheart, R. H. Scheller and J. E. Rothman, Cell, 1993, 3, 409-418.

63 F. Gaven, P. Marin and S. Claeysen, J. Visualized Exp., 2014, 91, e51751.

64 E. V. Mosharov and D. Sulzer, Nat. Methods, 2005, 2, 651658. 